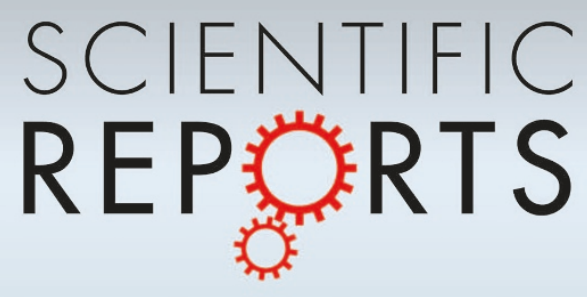

OPEN

SUBJECT AREAS:

LAB-ON-A-CHIP

ASSAY SYSTEMS

BIOLOGICAL PHYSICS

BIOMEDICAL ENGINEERING

Received

3 June 2013

Accepted

18 October 2013

Published

5 November 2013

Correspondence and requests for materials should be addressed to J.J.S. (schmidł@seas. ucla.edu)

\title{
Hydrogel-Stabilized Droplet Bilayers for High Speed Solution Exchange
}

\author{
Shiv A. Acharya', Alexander Portman', Carl S. Salazar² \& Jacob J. Schmidt' \\ 'Department of Bioengineering, University of California, Los Angeles, CA, 90095-1600, U.S.A., ${ }^{2}$ Librede Inc., Sherman Oaks, CA, \\ 91403.
}

\begin{abstract}
Many applications utilizing artificial lipid bilayers require the ability to exchange the bilayer's solution environment. However, because of the instability of the bilayer, the rate of solution exchange is limited, which significantly hinders the measurement rate and throughput. We have developed an artificial bilayer system that can withstand high flow speeds, up to $2.1 \mathrm{~m} / \mathrm{s}$, by supporting the bilayer with a hydrogel. We demonstrated the ability to measure during flow by measuring the conductance of gramicidin-A channels while switching between solutions of two different compositions, recording a time to measure $90 \%$ change in current of approximately 2.7 seconds at a flow rate of $0.1 \mathrm{~m} / \mathrm{s}$. We also demonstrated a potential application of this system by measuring the conductance modulation of the rat TRPM8 ion channel by an agonist and antagonist at varying concentrations, obtaining 7-point $\mathrm{IC}_{50}$ and $\mathrm{EC}_{50}$ values in approximately 7 minutes and 4 -point values within 4 minutes.
\end{abstract}

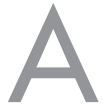
rtificial lipid bilayer membranes are well established for fundamental physiological studies of ion channels $s^{1,2}$ as well as technological applications including sensing ${ }^{3}$, drug potency measurement ${ }^{4-7}$, and potentially DNA sequencing. ${ }^{8}$. In many of these applications, it is often desirable to exchange the solution surrounding the bilayer during measurement to halt ion channel incorporation for single channel studies, to introduce analyte solutions for sensing, or to measure changes in ion channel conductance with changing pharmaceutical concentrations.

Solution exchange for freestanding lipid bilayer membranes can be problematic, as the membranes are fragile, deforming or rupturing in the presence of the small transmembrane pressure differences ${ }^{9}$ that can result from flowing solutions ${ }^{10-12}$. As a result, traditional bilayer solution perfusion is limited to low flow rates, which result in complete exchange of the surrounding solution in timescales on the order of minutes ${ }^{13-15}$. Several recent papers have described microfluidic systems capable of exchanging the surrounding solution in $10-100$ seconds ${ }^{10-12}$. With one of these systems, we measured the potency of drugs for the TRPM8 and hERG ion channels in lipid bilayers by measuring the ion channel conductance while solutions containing increasing drug concentrations were introduced adjacent to one side of the bilayer ${ }^{4,5}$. Total measurement time for 5 different concentrations was approximately 30 to 50 minutes ${ }^{5}$, and measurement of 8 different concentrations required approximately 80 minutes $^{4}$ due in part to the slow rate of solution perfusion tolerable by the bilayer.

Although solid-supported lipid bilayers are robust and can withstand high solution flow rates ${ }^{16}$, they are unable to support application of constant voltages or measurement of direct currents needed for most ion channel conductance studies. These are possible with hydrogel-supported membranes; previously we have shown that hydrogel-supported membranes have increased tolerance to transmembrane pressure and greater longevity ${ }^{9,17}$. Others have shown production of hydrogel bilayer "chips"18,19. Most relevant to this work, bilayers formed through contact of lipid monolayers (in some contexts also called droplet interface bilayers ${ }^{20-22}$ ) have also been shown to be compatible with hydrogel support ${ }^{23-26}$.

In this work, we demonstrate a lipid bilayer system compatible with high speed fluid exchange. We created a lipid bilayer through contact of a lipid monolayer formed at an oil/aqueous interface to a lipid monolayer formed at an oil/hydrogel interface. This contact area was masked with an aperture cut from a plastic film to help stabilize bilayer area during flow of the aqueous solution ${ }^{11}$. We found that the hydrogel allowed the bilayer to tolerate flow of the aqueous solution at flow speeds up to $2.1 \mathrm{~m} / \mathrm{s}$ without rupture. With these flow-stabilized bilayers, we measured the conductance of gramicidin-A channels during flow of solutions with different conductivity to precisely determine the timescale over which the solution is completely changed. Finally, we demonstrated a potential application of this device for ion channel drug potency measurements by measuring the conductance modulation of TRPM8 ion channels following rapid exchange of several solutions containing increasing drug concentrations, obtaining data for drug $\mathrm{IC}_{50}$ and $\mathrm{EC}_{50}$ values in $<4$ minutes. The platform's simplicity, combined 
with its compatibility with automation and parallelization ${ }^{27,28}$, indicate its potential as a tool for ion channel studies and screening applications.

\section{Results}

In our recent work developing automatable, parallelizable droplet bilayer platforms $s^{5,28}$, an aqueous droplet attached to a movable electrode composed the upper aqueous solution of the lipid membrane environment. In this work, this droplet was replaced with an agarose hydrogel droplet protruding from a pipette tip. Fabrication of these agarose droplets was simple and compatible with high throughput parallel fluid handling hardware. Once made, the hydrogels could be stored in buffer at $4^{\circ} \mathrm{C}$ for weeks with no measureable difference in results. To investigate the effects of solution flow on the hydrogelstabilized droplet bilayer membrane, we measured bilayer electrical resistance as the flow rate of the adjacent solution was increased. The solution was continuously flowed through the lower channel of the chip while the flow rate was increased every 2 seconds until the syringe pump reached its maximum drivable flow rate or until bilayer failure, indicated by a sudden, large decrease in measured resistance. Gel-supported bilayers measured in chips with a $4 \mathrm{~mm}$ lower channel width showed no change in resistance during flow for all pump flow rates, up to the pump's maximum, $69.5 \mathrm{~mL} / \mathrm{min}$. For the $4 \mathrm{~mm}$ wide lower channel, this flow rate corresponds to a flow speed in the lower channel of $0.32 \mathrm{~m} / \mathrm{s}$. To measure the effects of greater flow speeds, we produced chips with smaller lower channel widths ( $2 \mathrm{~mm}, 1 \mathrm{~mm}$, and $0.5 \mathrm{~mm}$ ) so that a given flow rate resulted in increased flow speeds through the lower channel. Even with these smaller channels, we were able to perfuse solution at the maximum drivable flow rate in the $2 \mathrm{~mm}$ and $1 \mathrm{~mm}$ width lower channels with no bilayer rupture, corresponding to $0.69 \mathrm{~m} / \mathrm{s}$ and $1.37 \mathrm{~m} / \mathrm{s}$ flow speeds. We could only observe failure of the gel-supported DIBs with a $500 \mu \mathrm{m}$ width lower chamber at flow speeds greater than $2.1 \mathrm{~m} / \mathrm{s}$; failure was indicated by an immediate, huge reduction in electrical resistance. At high flow speeds $(>0.2 \mathrm{~m} / \mathrm{s})$ the measured electrical current in response to applied potential began to decrease with increasing flow speed, possibly due to increasing streaming potential. As a result, for the remaining measurements, the flow speed was limited to $0.1 \mathrm{~m} / \mathrm{s}$, where this effect was not observed.

Having demonstrated the stability of the gel supported droplet bilayers over a wide range of flow speeds, we investigated ion channel measurement during solution exchange using gramicidin- $\mathrm{A}(\mathrm{gA})$ as a model ion channel. We prepared agarose gel electrodes with $1 \mathrm{M} \mathrm{KCl}$ buffer and used them to make DPhPC bilayers with $3 \mathrm{fg} / \mathrm{mL} \mathrm{gA} \mathrm{in}$ chips with a $1 \mathrm{~mm}$ wide lower channel. Following bilayer formation, $100 \mathrm{mV}$ was applied and steady gA currents were measured with an

A

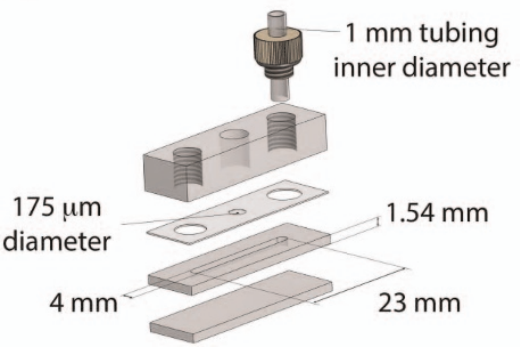

average current of approximately $180 \mathrm{pA}$ and fluctuations of $\sim$ $40 \mathrm{pA}$, due to the aggregate random binding and unbinding of many conducting gA dimers ${ }^{29}$. Close examination of the measured currents showed single channel steps of $2.37 \pm 0.28 \mathrm{pA}(\mathrm{N}=40)$ (Supplementary Information). Flow of $1 \mathrm{M} \mathrm{KCl}$ buffer through the lower channel at $5 \mathrm{~mL} / \mathrm{min}(0.1 \mathrm{~m} / \mathrm{s}$ flow speed $)$ affected neither the magnitude of measured current nor the magnitude of the aggregated dimer current fluctuations. The observed average currents and fluctuation size also remained consistent when the flow was stopped and restarted with the syringe pump.

In another experiment exploring rapid exchange of different solutions, a solenoid valve was triggered to switch the perfused solutions between $100 \mathrm{mM} \mathrm{KCl}, 900 \mathrm{mM}$ TEA-Cl and $1 \mathrm{M} \mathrm{KCl}$ while applying $-80 \mathrm{mV}$ and measuring the resultant gA current (Figure 2). The 900 mM TEA-Cl maintained the osmotic balance and chloride concentration across the bilayer while replacing gA-conductive $\mathrm{K}^{+}$ions with non-conductive $\mathrm{TEA}^{30}$, producing a large difference in concentration of conductive $\mathrm{K}^{+}$ions across the bilayer after it was perfused. The solution was exchanged three times, producing four separate intervals where the measured current alternated between two distinct levels. $-142 \pm 5.0 \mathrm{pA}$ and $-9 \pm 1.2 \mathrm{pA}$, measured with $1 \mathrm{M} \mathrm{KCl}$ and $100 \mathrm{mM} \mathrm{KCl}$ in the lower solutions, respectively, each averaged over the two seconds preceding the actuation of the solenoid valve. Control experiments, in which the same solutions were switched during measurement of a gel-supported bilayer containing no ion channels, showed an unchanging small measured current $(<5 \mathrm{pA})$ for all solutions over the course of the entire experiment. The difference in gA conductance in the two exchanged solutions was also seen at the single channel level. After filtering the data with a $160 \mathrm{~Hz}$ low pass 8-Pole Bessel filter and a $60 \mathrm{~Hz}$ notch filter, $1.85 \pm 0.25 \mathrm{pA}$ $(\mathrm{N}=38)$ steps could be identified during measurement in the $1 \mathrm{M}$ $\mathrm{KCl}$ solution, while $0.38 \pm 0.11 \mathrm{pA}(\mathrm{N}=26)$ steps were identified from the currents measured in $1 \mathrm{M} \mathrm{KCl} / 100 \mathrm{mM} \mathrm{KCl}-900 \mathrm{mM}$ TEA-Cl ( $-80 \mathrm{mV}$ potential applied throughout) (Supplementary Information).

The measured current shown in Figure 2 exhibited a consistent, approximately $750 \mathrm{~ms}$ lag between the triggering of the valve and the start of the change in measured current. This lag corresponds to the time required to pump the dead volume of solution in the tubing between the solenoid valve and the chamber; the dead volume is approximately $65 \mu \mathrm{L}$, which would take $780 \mathrm{~ms}$ to transfer at a flow rate of $5 \mathrm{~mL} / \mathrm{min}$, matching the observed time well. Following this initial time lag, the time required for the current to reach $90 \%$ of its steady state value from the start of its change was approximately 2.7 seconds.

We also simulated the exchange of varying ionic strength solutions through the lower chamber using COMSOL. The model geometry

B

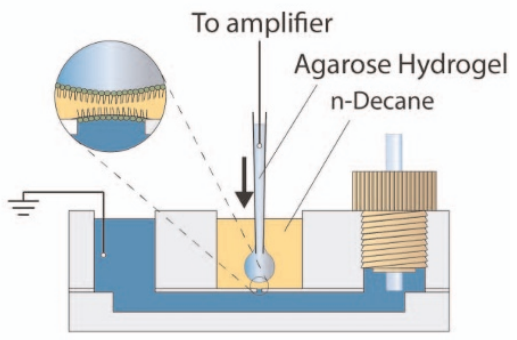

Figure $1 \mid$ Chip schematic and use. (A) Exploded view. The chip consists of four acrylic pieces: one top piece and two bottom pieces sandwich a thin middle partition containing a $175 \mu \mathrm{m}$ diameter center aperture. The two upper holes are connected through the partition to a single channel in the lower acrylic piece and are accessible from the top. (B) Cross-sectional diagram of assembled chip in use. The lower channel is filled with liposome solution and $\mathrm{n}$-decane is added to the center well. The gel-tipped electrode is dipped in liposome solution and lowered into n-decane. Following lipid monolayer formation, a lipid bilayer is formed upon contact of the gel tip to the lower aqueous solution, bounded by the partition. Transmembrane currents are recorded by an amplifier connected to the gel-tipped electrode and counter-electrode in the outer well. Connection of the lower channel fluidic inlet to a syringe pump allows exchange of the lower aqueous solution. 
A

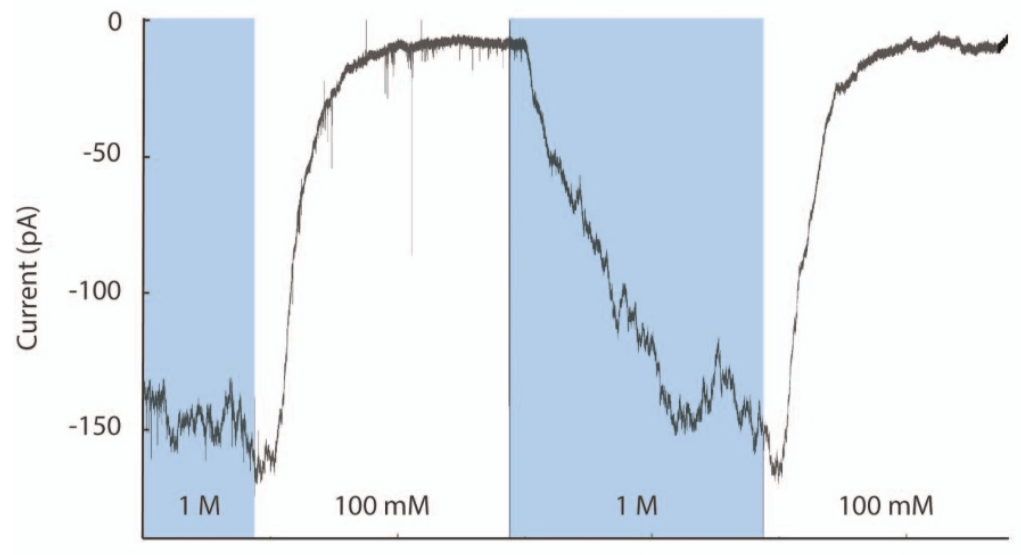

0

10

20

30

Time (seconds)

B

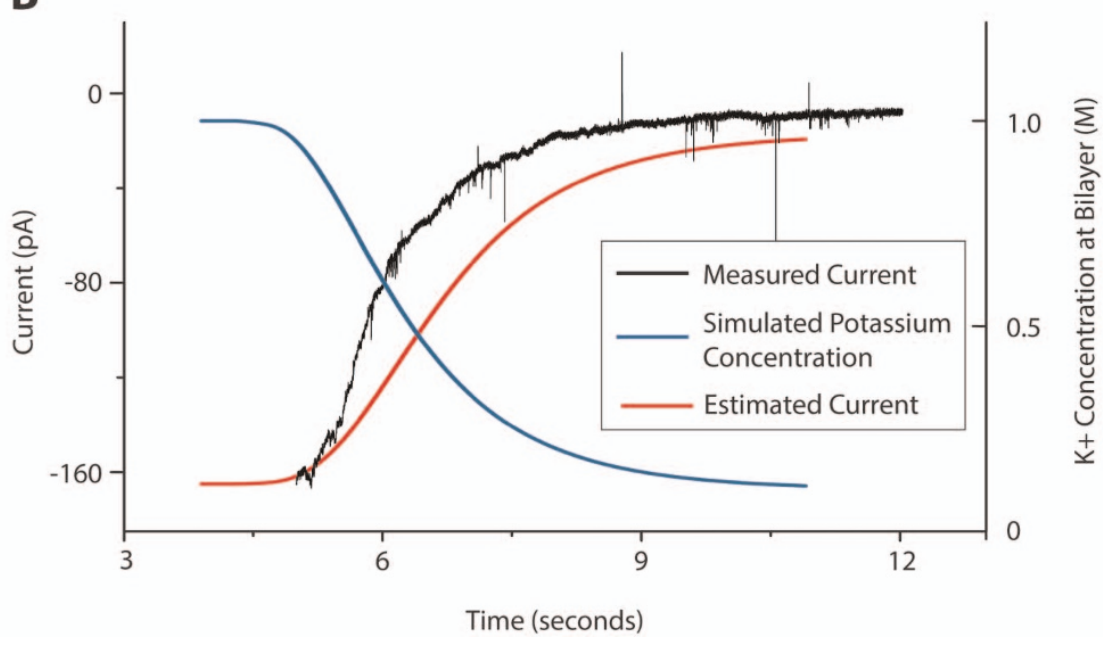

Figure $2 \mid$ (A) Unfiltered measurement of gramicidin-A conductance during repeated exchange of two different solutions. Buffered solutions containing $1 \mathrm{M} \mathrm{KCl}$ (10 mM HEPES, pH 7.2) and $100 \mathrm{mM} \mathrm{KCl,} 900 \mathrm{mM}$ TEA-Cl (10 mM HEPES, pH 7.2) were alternately flowed through the measurement chamber during application of $-80 \mathrm{mV}$ transmembrane potential to a gel-supported bilayer containing gramicidin-A. The times corresponding to activation of a solenoid valve that switched between the two solutions are indicated by the shaded areas; three transitions are shown. (B) A COMSOL model simulated the $\mathrm{K}^{+}$concentration during exchange of the $1 \mathrm{M}$ solution for the $100 \mathrm{mM}$ solution (blue curve). Based on this simulated concentration and the assumption that the number of conducting gA channels remained constant, the gramicidin current was estimated using the GHK equation (red curve, see text). Measured current data from (A) is overlaid (black).

was designed to match the geometry of the chip without tubing. The laminar flow physics module was used to calculate flow through the system, using a flow velocity inlet condition and a zero pressure outlet condition. All other boundaries were given no-slip conditions. Particle tracing was calculated by the transport of diluted species physics module, defining convection of particles by the steady-state solution of the laminar flow calculation, and calculating diffusion based on a diffusion constant of $1.9 \times 10^{-9} \mathrm{~m}^{2} / \mathrm{sec}^{31}$. Initial particle concentration was defined to be $10^{-5} \mathrm{~mol} / \mathrm{m}^{3}$ (1 M concentration) for the entire geometry except for the inlet boundary, which was given a particle concentration of $10^{-6} \mathrm{~mol} / \mathrm{m}^{3}(0.1 \mathrm{M})$, to match the conditions of the experiment shown in Figure $2 a$. Figure $2 b$ shows the simulated $\mathrm{K}^{+}$concentration as a function of time for 7 seconds following addition of the exchanged solution at $18.4 \mu$ s time steps.

Having demonstrated the ability of our bilayer apparatus to support high speed flow and solution exchange during ion channel measurement, we explored application of this system to rapid measurement of the potency of ion channel targeting drugs. We selected TRPM8, a mammalian ion channel responsible for cold sensing and associated with prostate and colon cancers ${ }^{32-35}$, for these experiments based on our previous work measuring its drug-modulated conductance in droplet bilayers ${ }^{4}$. In that previous work, TRPM8 currents were measured during the stepwise addition of menthol, an agonist compound, or 2-APB, an antagonist; these experiments required 50 to 80 minutes to complete. The length of these experiments was due in part to the sensitivity of the droplet bilayers to perturbation of surrounding solution, which necessitated extremely slow solution perfusion.

We made gel supported droplet bilayers using $200 \mathrm{~nm}$ diameter 3:1 POPC: POPE liposomes containing TRPM8 in buffer MB (described in Materials and Methods) containing $2.5 \mu \mathrm{M} \mathrm{PIP}$. While $100 \mathrm{mV}$ was applied, the transmembrane current was recorded for one minute. A syringe pump loaded with a syringe containing $\mathrm{MB}$ and a low concentration of drug was connected to the fluid inlet and activated to introduce the solution at $0.1 \mathrm{~m} / \mathrm{s}$ flow speed ( $20 \mathrm{~mL} / \mathrm{min}$ with the $4 \mathrm{~mm}$-wide channel) for 10 seconds after which it was stopped. The conductance was measured for 30 seconds; during this time, the syringe in the syringe pump was exchanged with a syringe containing a higher concentration of the drug and the process was repeated. 
Experiments measuring TRPM8 currents in the presence of varying concentrations of menthol and 2-APB were performed three times each on separate bilayers (Figure 3 ). $\mathrm{EC}_{50}$ and $\mathrm{IC}_{50}$ values were determined by fitting the measured current data as a function of drug concentration to the Hill equation using Origin 8.5 software (OriginLab). The $\mathrm{EC}_{50}$ values for menthol found through these experiments were $60.9 \mu \mathrm{M}, 45.4 \mu \mathrm{M}$, and $70.7 \mu \mathrm{M}$, similar to literature values of 53 to $101 \mu \mathrm{M}^{36-38}$. The $\mathrm{IC}_{50}$ values found for 2 -APB were $1.66 \mu \mathrm{M}, 2.69 \mu \mathrm{M}$, and $3.31 \mu \mathrm{M}$, with literature values of 1.5 to $12 \mu \mathrm{M}^{4,39,40}$. The time required to complete each set of measurements was related to the number of different concentrations measured, with four concentrations requiring $198-241 \mathrm{sec}$ and eight concentrations $432 \mathrm{sec}$.

\section{Discussion}

The presence of the hydrogel on one side of the bilayer greatly increased its tolerance to solution flow, with failure only occurring at flow speeds $>2.1 \mathrm{~m} / \mathrm{s}$, much greater than the maximum flow speed seen in our previous work $\left(2.5 \times 10^{-4} \mathrm{~m} / \mathrm{s}\right)$ using a highly similar system without a hydrogel ${ }^{11}$. This speed is also greater than other recent reports measuring solution perfusion of droplet bilayers in microfluidic devices ${ }^{10,12}$.

We hypothesize that the tolerance of the hydrogel supported membrane to increased flow speeds is a result of the increased tolerance of hydrogel supported membranes to transmembrane pressure differences, which we showed previously ${ }^{9}$. The hydrogel opposes deformation of the bilayer and separation from its supporting aperture. This hypothesis is consistent with the low flow rates observed in our and others' previous reported work ${ }^{10,11}$. In these reports, solution exchange was driven with a syringe pump where the fluidic outlet was exposed to atmosphere. In such an apparatus, assuming a bilayer chamber with symmetric inlet and outlet fluidic connections, the hydrostatic pressure at the bilayer would be half of the inlet pressure. This inlet pressure would increase proportionally

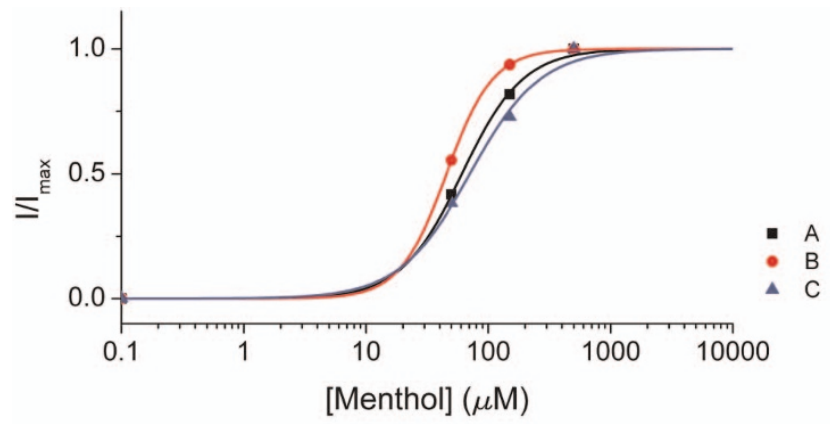

\begin{tabular}{ccc} 
& \multicolumn{1}{c}{$\mathrm{EC}_{50}$} & $60.9 \pm 2.44 \mu \mathrm{M}$ \\
\cline { 2 - 3 } & Acquisition Time & $3 \mathrm{~min} 32 \mathrm{sec}$ \\
$\mathrm{n}$ & $\mathrm{EC}_{50}$ & $45.4 \pm 0.288 \mu \mathrm{M}$ \\
\cline { 2 - 3 } $\mathrm{C}$ & Acquisition Time & $3 \mathrm{~min} 18 \mathrm{sec}$ \\
& $\mathrm{EC}_{50}$ & $70.7 \pm 6.63 \mu \mathrm{M}$ \\
\cline { 2 - 3 } $\mathrm{C}$ & Acquisition Time & $3 \mathrm{~min} 2 \mathrm{sec}$
\end{tabular}

to the flow rate, according to Poiseuille's Law. In these systems, the pressure on the other side of the bilayer is fixed because that side of the bilayer is open to atmosphere. Therefore the transmembrane pressure would also increase proportionally to the flow rate. This hypothesis also explains the higher flow rates of bilayer solution exchange in the work of Tsuji et al. through the use of a push-pull syringe pump ${ }^{12}$. The push-pull mechanism of the pump imposes a positive pressure on the inlet of the channel and a matching negative pressure on the outlet of the channel, resulting in a fluid pressure close to atmospheric pressure near the bilayer and therefore a greatly reduced pressure gradient across the bilayer. Therefore, higher flow rates can be tolerated before bilayer rupture. Our approach offers two advantages: first, we were able to obtain higher flow rates. Second, a push-only solution exchange system is much more amenable to parallelization and automation such as with multi-channel robotic pipette heads.

Although the rate of fluid flow through the measurement chamber may be easily controlled and determined by the syringe pump, ascertaining the exact time for which the measurement solution is fully exchanged is complicated by the static fluid boundary layer at the chamber walls and the exact position of the bilayer relative to the flowing solution. Our measurements of gA during flow were intended to empirically monitor the progress of solution exchange by continuously measuring gA current as the measurement solution was switched between $1 \mathrm{M}$ and $100 \mathrm{mM}$ concentrations of the only conductive ion, potassium.

Two main effects were expected when switching between the lower solution from $1 \mathrm{M}$ to $100 \mathrm{mM}$ : 1) establishment of a Nernst potential from $0 \mathrm{mV}$ to $V_{N}=-\frac{k_{B} \mathrm{~T}}{e} \ln \frac{a_{\text {gel }}}{a_{\text {lower }}}=-54 \mathrm{mV}$, where $\mathrm{a}_{\text {gel }}$ is the $1 \mathrm{M} \mathrm{K}^{+} \operatorname{activity}^{41}(0.6 \mathrm{M})$ and $a_{\text {lower }}$ is the $1 \mathrm{M} \mathrm{K}^{+}$activity $^{41}$ $(0.074 \mathrm{M})$ and 2$)$ decreased $\mathrm{K}^{+}$conductivity in the lower solution. Since we constantly applied $-80 \mathrm{mV}$ in these measurements, we expected both of these effects to result in a lower measured current.

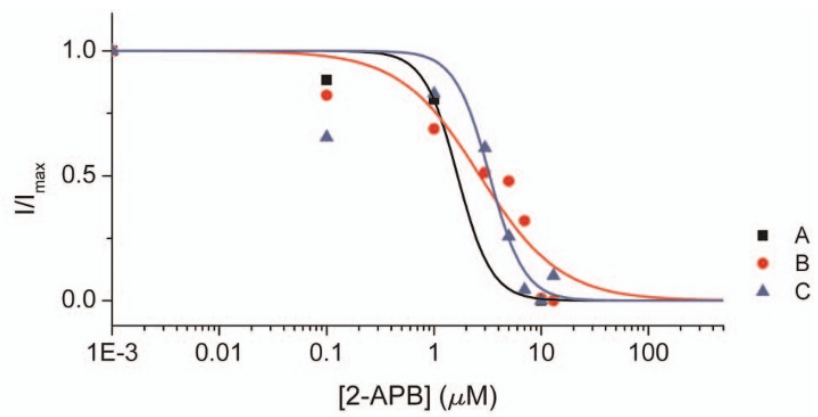

\begin{tabular}{|c|c|c|}
\hline \multirow{2}{*}{ A } & $\mathrm{IC}_{50}$ & $1.66 \pm 1.57 \mu \mathrm{M}$ \\
\hline & Acquisition Time & $4 \mathrm{~min} 1 \mathrm{sec}$ \\
\hline \multirow{2}{*}{ B } & $\mathrm{IC}_{50}$ & $2.69 \pm 0.758 \mu \mathrm{M}$ \\
\hline & Acquisition Time & $7 \mathrm{~min} 12 \mathrm{sec}$ \\
\hline \multirow{2}{*}{ C } & $\mathrm{IC} \mathrm{C}_{50}$ & $3.31 \pm 0.648 \mu \mathrm{M}$ \\
\hline & Acquisition Time & $7 \mathrm{~min} 12 \mathrm{sec}$ \\
\hline
\end{tabular}

Figure 3 TRPM8 activation and inhibition by menthol and 2-APB, respectively. (Left) In three separate experiments (A), (B), (C), menthol was perfused into the chip in increasing concentrations $(0,50,150,500 \mu \mathrm{M})$ and the current was measured for 30 seconds while applying $100 \mathrm{mV}$. These currents were averaged and normalized to the current measured at $500 \mu \mathrm{M}$ menthol. $\mathrm{EC}_{50}$ values were obtained from fits of the data to the Hill equation. (Right) In three experiments, 2-APB was perfused into the chip in increasing concentrations in solutions also containing $500 \mu \mathrm{M}$ menthol. Currents were measured similarly to the previous menthol experiments (Left). In one experiment (A), the 2-APB concentrations measured were $0,0.1,10,13 \mu \mathrm{M}$. In two experiments (B), (C) the concentrations were $0,0.1,1,3,5,7,10,13 \mu \mathrm{M}$. IC $_{50}$ values were obtained from fits of the data to the Hill equation. Acquisition times represent the total time from initiation to conclusion of all measurements. 
We can quantitatively estimate the expected current level by using the Goldman-Hodgkin-Katz current equation ${ }^{42}$ for potassium:

$$
\begin{aligned}
I_{K} & =\frac{e^{2} 1000 N_{A} P_{K}}{k_{B} T} V\left[\frac{a_{\text {lower }} e^{-\frac{e V}{k_{B} T}}-a_{g e l}}{e^{-\frac{e V}{k_{B} T}}-1}\right] \\
& =G_{K}\left(a_{\text {lower }}\right) V\left[\frac{e^{-\frac{e V}{k_{B} T}}-\frac{a_{g e l}}{a_{\text {lower }}}}{e^{-\frac{e V}{k_{B} T}}-1}\right]
\end{aligned}
$$

where $\mathrm{V}$ is the potential applied between the tip and lower channel, $\mathrm{k}_{\mathrm{B}}$ is $1.38 \times 10^{-23} \mathrm{~J} / \mathrm{K}$, T is the temperature, e is $1.602 \times 10^{-19} \mathrm{C}, \mathrm{N}_{\mathrm{A}}$ is $6.02 \times 10^{23}$, and $\mathrm{P}_{\mathrm{K}}$ is the permeability of the bilayer to potassium. After factoring $a_{\text {lower }}$ from the bracketed expression, we define $\mathrm{G}_{\mathrm{K}}\left(\mathrm{a}_{\text {lower }}\right)$ as $G_{K}=\frac{e^{2} 1000 N_{A} P_{K}}{k_{B} \mathrm{~T}} a_{\text {lower }}$. Therefore, when the $\mathrm{K}$ concentrations in the gel and lower channel are both $1 \mathrm{M}$, $I_{K_{1 M}}=G_{K}\left(a_{1 M}\right) V$. The single channel gA current steps measured with $1 \mathrm{M} \mathrm{KCl}$ present on both sides of the bilayer were $1.85 \mathrm{pA}$, a conductance of $23 \mathrm{pS}$ at $-80 \mathrm{mV}$, consistent with $\sim 21 \mathrm{pS}$ of previous work ${ }^{43}$. Upon exchanging the lower solution to $100 \mathrm{mM} \mathrm{KCl}$, $0.38 \mathrm{pA}$ steps were identified. With the $\mathrm{K}$ concentration in the gel and lower solutions of $1 \mathrm{M}$ and $0.1 \mathrm{M}$, respectively, Eq. 1 becomes $\mathrm{I}_{\mathrm{K}}$ $=\mathrm{G}_{\mathrm{K}}\left(\mathrm{a}_{\text {lower }}\right) * \mathrm{~V} * 0.66$, and the gA conductance we obtain for these steps is $7.2 \mathrm{pS}$, comparable to $\sim 9 \mathrm{pS}$ measured at $100 \mathrm{mV}$ in $0.1 \mathrm{M}$ $\mathrm{KCl}$ by Busath et al. in DPhPC bilayers ${ }^{43}$.

In Figure 2, the current measured before the actuation of the solenoid at $\mathrm{t}=4.5$ seconds was $-165 \mathrm{pA}$, corresponding to a conductance $\mathrm{G}_{\mathrm{K}}$ of $2063 \mathrm{pS}$, approximately 90 channels. The current is stabilized following the solution exchange at approximately $9 \mathrm{pA}$, a conductance of $170 \mathrm{pS}$ from Equation 1. From our single channel conductance measurement of $0.38 \mathrm{pA}, 9 \mathrm{pA}$ is approximately 24 channels, a significant reduction from the 90 estimated prior to the solution exchange. A reduction in the number of channels is consistent with previous reports of a reduction in $\mathrm{gA}$ 's dimer equilibrium constant with a reduction in electrolyte concentration ${ }^{44}$.

The time course of the simulated concentration can be related to the measured current through application of the simulated data to Equation 1. The ionic activity was found from the concentration using the data from Hamer and $\mathrm{Wu}^{41}$, and the gA single channel conductance in DPhPC for different $\mathrm{KCl}$ concentrations was found from Busath et al. ${ }^{43}$. The data from Busath et al. was measured in symmetric solutions and without TEA-Cl, and so it may not be fully applicable here. The activity and gA conductance was input into Equation 1 for calculated estimates of the gA current as a function of time (Figure $2 b$ ). The measured current appears to decrease faster and plateau at a smaller magnitude than the predicted current. As the predicted current assumes that the number of conducting channels in the bilayer is constant, this could be explained by our observation of the number of conducting channels decreasing during the solution exchange. The time for the simulated concentration to fall $90 \%$ from $1 \mathrm{M}$ to $0.1 \mathrm{M}$ is 3.7 seconds, the same time required for the calculated current to rise $90 \%$ from $-165 \mathrm{pA}$ to $-19.6 \mathrm{pA}$. The measured current required only 2.5 seconds to rise $90 \%$ from $-165 \mathrm{pA}$ to $-7.3 \mathrm{pA}$. The faster than predicted decrease in current is also consistent with the decreased number of conducting channels measured in the lower concentration.

Tsuji and coworkers recently reported solution exchange surrounding a bilayer using a push-pull syringe pump and measured the exchange time through fluorescence measurement as the fluorophore was removed from the observed solution ${ }^{12}$. Their simulations of the non-laminar flow showed that the solution adjacent to the bilayer flowed at approximately $1 / 5$ of the pumped rate, indicating the effects of the boundary layer to effectively slow the flow near the bilayer. This slowed flow resulted in a time for the fluorescence decrease to $1 \%$ of its initial value of 16.5 seconds.
For repeated ion channel measurement in a series of exchanged solutions, the total time required will be the sum of the time for solution exchange and the time for the measurements themselves. In the series of TRPM8 measurements described here, the solution flow speed was $0.1 \mathrm{~m} / \mathrm{s}$ which resulted in approximately $4.5 \mathrm{sec}$ for complete solution exchange (Figure 2). To ensure that the solutions were entirely exchanged, we flowed the solutions for $10 \mathrm{sec}$ before deactivating the pump. The following 30 second measurement time was also conservatively chosen to minimize statistical uncertainty in determination of the average current. Taken together and including the time required for fluid handling, the total measurement time per drug concentration was at least 50 seconds, requiring approximately 200 and 400 seconds for four and eight measured concentrations respectively. This is a good improvement over previous measurements of concentration-dependent drug interactions with ion channels in lipid bilayers ${ }^{4-7}$, in which slow rates of exchange of measurement solution were primary factors contributing to assay times greater than one hour.

In conclusion, we have demonstrated that hydrogel-supported lipid bilayers are sufficiently robust to withstand the exchange of adjacent solution at high flow rates $(>2 \mathrm{~m} / \mathrm{s})$ without bilayer rupture. Rapid exchange of the measurement solution enables increased measurement efficiency and throughput. Our bilayer chip and measurement apparatus allowed electrical measurement of the bilayer and ion channels during flow. Using this apparatus, we were able to measure the concentration-dependent inhibition of the conductance of the ion channel TRPM8, obtaining $\mathrm{IC}_{50}$ and $\mathrm{EC}_{50}$ values in minutes, versus previously reported times on the order of an hour.

As with our previous work, the upper and lower halves of the bilayer are accessible from the top of the chip for fluid addition and bilayer formation and measurement ${ }^{4,5}$. The gel-tipped electrodes integrate bilayer formation and measurement and require only simple vertical translation to begin the assay. This step, and the addition of the solutions to the chip, can be easily automated ${ }^{28}$ and paralle$\operatorname{lized}^{27}$ with the construction of repeated arrays of the basic chip design shown in Figure 1. The gel-tipped electrodes can be massprepared in advance of the measurement and stored until use. These advantages and the short assay time give this platform potential for high throughput measurement and screening.

\section{Methods}

Unless otherwise noted, all reagents and chemicals were purchased from Sigma Aldrich.

Bilayer chip. The bilayer chip design (Figure 1A) was adapted from previous work ${ }^{5,28}$ The chip was laser cut from acrylic sheets (McMaster Carr, Techplast). The chip top was $1 / 4$ " thick with three collinear holes $5 \mathrm{~mm}$ in diameter. The outer holes were tapped with 10-32 size threads to accommodate fluidic connections. The bottom of the chip consisted of a $23 \mathrm{~mm}$ long channel ranging from 0.5 to $4 \mathrm{~mm}$ in width (depending on the experiment) formed from two 1/16" thick acrylic sheets. Between the chip top and bottom was a $250 \mu \mathrm{m}$ thick acrylic sheet containing three collinear holes with center positions matching those of the chip top. Two peripheral holes had $5 \mathrm{~mm}$ diameter matching the inlet/outlet ports of the chip top and a $175 \mu \mathrm{m}$ diameter hole aligned with the central hole of the chip top. The $175 \mu \mathrm{m}$ diameter hole was cut at the center of a $2.5 \mathrm{~mm}$ diameter region in which the acrylic was thinned using the laser to $100 \pm 2 \mu \mathrm{m}$ thickness, as measured by a digital micrometer (Mitutoyo). Once assembled, the lower channel is accessible through the peripheral holes in the chip top and connects to the upper part of the center well through only the $175 \mu \mathrm{m}$ diameter hole. After assembly, the chip was glued using Weld-On Type 4 (SCI Grip).

Bilayer formation. $200 \mathrm{~nm}$ diameter liposomes, composed of $250 \mu \mathrm{g} / \mathrm{mL}$ diphytanoylphosphatidylcholine (DPhPC) or $250 \mu \mathrm{g} / \mathrm{mL}$ of $3: 1$ (w:w) 1-palmitoyl2-oleoyl-sn-glycero-3-phosphocholine (POPC) and 1-palmitoyl-2-oleoyl-snglycero-3-phosphoethanolamine (POPE) (Avanti Polar Lipids), were prepared by extrusion through a $200 \mathrm{~nm}$ filter in measurement buffer MB (150 mM KCl, $0.2 \mathrm{mM}$ $\mathrm{MgCl}_{2}$ (10 mM HEPES, $\left.\mathrm{pH} 7.2\right)$ or $1 \mathrm{M} \mathrm{KCl}(10 \mathrm{mM}$ HEPES, $\mathrm{pH}$ 7.2)). The chip was prepared for use by filling the lower chamber through the peripheral wells with $200 \mu \mathrm{L}$ of the liposome solution followed by addition of $80 \mu \mathrm{L}$ of $\mathrm{n}$-decane to the upper central well (Figure 1B). $1.35 \mu \mathrm{L}$ of the liposome solution was deposited onto an agarose gel bead (described below) and the gel bead was lowered into the central well until it was completely submerged in n-decane (Figure 1B). After a waiting period 
of 5 minutes to allow lipid monolayers to form, the gel bead was lowered to contact the $175 \mu \mathrm{m}$ diameter aperture where the bilayer formed once the monolayers contacted.

Sessile agarose droplet. A $1 \%(\mathrm{w} / \mathrm{v})$ solution of low melting point agarose (Invitrogen) was prepared in $\mathrm{MB}$, except during experiments varying ionic strength, when it was prepared in $1 \mathrm{M} \mathrm{KCl}(10 \mathrm{mM}$ HEPES, $\mathrm{pH}$ 7.2). The solution was warmed to $50^{\circ} \mathrm{C}$ and approximately $100 \mu \mathrm{L}$ of it was drawn into a $200 \mu \mathrm{L}$ gel-loading pipette tip (VWR). The solution was slowly dispensed out of the pipette tip to form a $\sim 3 \mu \mathrm{L}$ sessile droplet at the end of the tip, which was cooled to the gel state. The pipette tip was then backfilled with $\mathrm{MB}$ or $1 \mathrm{M} \mathrm{KCl}$ and stored with the agarose sessile droplet immersed in the same solution at $4{ }^{\circ} \mathrm{C}$. Formation of gel tipped electrodes in this way was easy and rapid, and they were storable for extended periods of time at $4{ }^{\circ} \mathrm{C}$.

Electrophysiological measurement. $\mathrm{Ag} / \mathrm{AgCl}$ electrodes were inserted into the top of the pipette gel tip and the outlet port of the bilayer chip and connected to an Axopatch 200B amplifier (Axon Instruments), which applied a $1 \mathrm{kHz}$ Bessel filter to the amplified currents. The resulting signals were digitized at $10 \mathrm{kHz}$ (Digidata 1440A, Axon Instruments) and further filtered and analyzed with Clampfit 10 software (Axon Instruments).

Gramicidin-A channels were diluted to $3 \mathrm{fg} / \mathrm{mL}$ in a solution of DPhPC liposomes in $1 \mathrm{M} \mathrm{KCl}$ (10 mM HEPES, $\mathrm{pH}$ 7.2) and used for bilayer formation, as previously described. Rat TRPM8 ion channels were purified and reconstituted into $3: 1$ POPC: POPE liposomes at a $1: 1,000$ protein : lipid ratio $(\mathrm{w}: \mathrm{w})$, as previously reported $^{4,45} .10 \mathrm{mg} / \mathrm{mL}$ proteoliposome stock solutions were diluted to $1 \mathrm{mg} / \mathrm{mL}$ prior to measurement in $\mathrm{MB}$ and $2.5 \mu \mathrm{M}$ phosphatidylinositol-4,5-bisphosphate $\left(\mathrm{PI}(4,5) \mathrm{P}_{2}\right.$, Avanti Polar Lipids), a compound required for TRPM8 activation ${ }^{45}$. All experiments were conducted at room temperature, approximately $21^{\circ} \mathrm{C}$

Solution exchange. $30 \mathrm{~mL}$ plastic syringes (Becton-Dickinson) were connected to the inlet hole of the bilayer chip by $1 \mathrm{~mm}$ inner diameter Teflon tubing (SigmaAldrich) and 10-32 size flangeless fittings (Sigma-Aldrich). The syringes were driven by a single syringe pump (KDS Legato 200, KD Scientific), controlled through Windows HyperTerminal command prompts to drive solution at variable rates through the bilayer chip's lower channel (Figure 1B). For experiments in which perfusion of two solutions was alternated, a system of four two-way solenoid valves directed and alternated flow from two syringes such that flow from one syringe went to the bilayer chip, and flow from the other syringe went into a waste container. In experiments in which more than two solutions were perfused into the chip, a solenoid valve was switched to direct flow from an external line to the syringe. The syringe was then filled with the appropriate perfusion solution, and the valve was switched back to direct flow toward the chip. Solenoid valve actuation was controlled was LabVIEW 9.2.1 software (National Instruments).

In experiments in which perfusion speed limits were explored, the solution used was MB. In experiments in which the composition of the lower aqueous solution was changed (Fig. 2), $1 \mathrm{M} \mathrm{KCl} \mathrm{(10} \mathrm{mM} \mathrm{HEPES,} \mathrm{pH} \mathrm{7.2)} \mathrm{and} 100 \mathrm{mM} \mathrm{KCl}, 900 \mathrm{mM}$ Tetraethylammonium Chloride (TEA-Cl) (10 mM HEPES, $\mathrm{pH} 7.2)$ buffer were used. During measurements of TRPM8 (Fig. 3), MB solutions containing varying concentrations of Menthol or 2-Aminoethoxydephenyl Borate (2-APB) were used.

Ion convection and diffusion modeling. COMSOL Multiphysics 4.2 a (COMSOL, Stockholm, Sweden) was used to model the solution flow through the lower chamber during exchange of $1 \mathrm{M} \mathrm{KCl}$ solution for $0.1 \mathrm{M} \mathrm{KCl}$. The Laminar Flow physics module was used to calculate flow through the system, using a flow velocity inlet condition and a zero pressure outlet condition. All other boundaries were given noslip constraints. Particle tracing was calculated by the Transport of Diluted Species physics module, defining convection of particles by the steady-state solution of the laminar flow calculation and calculating diffusion based on a diffusion constant of 1.9 $\times 10^{-9} \mathrm{~m}^{2} / \mathrm{sec}^{31}$. Initial particle concentration was defined to be $1 \mathrm{M}$ for the entire geometry except for the inlet boundary, which was given a particle concentration of $1 \mathrm{M}$ to match the transitions between shaded and unshaded regions in Figure 2.

1. Schindler, H. \& Quast, U. Functional acetylcholine receptor from Torpedo marmorata in planar membranes. Proc. Natl. Acad. Sci. USA. 77, 3052-3056 (1980).

2. Ion channel reconstitution, Miller, C. (ed.) (Plenum Press, 1986)

3. Bayley, H. \& Cremer, P. S. Stochastic sensors inspired by biology. Nature 413, 226-230 (2001).

4. El-Arabi, A. M., Salazar, C. S. \& Schmidt, J. J. Ion channel drug potency assay with an artificial bilayer chip. Lab Chip 12, 2409-2413, doi:10.1039/c2lc40087a (2012).

5. Portonovo, S. A., Salazar, C. S. \& Schmidt, J. J. hERG drug response measured in droplet bilayers. Biomed. Microdev. doi:10.1007/s10544-012-9725-9 (2012).

6. Syeda, R., Holden, M. A., Hwang, W. L. \& Bayley, H. Screening blockers against a potassium channel with a droplet interface bilayer array. J. Am. Chem. Soc. 130, 15543-15548 (2008).

7. Tao, X. \& MacKinnon, R. Functional analysis of Kv1. 2 and paddle chimera Kv channels in planar lipid bilayers.J. Mol. Biol. 382, 24-33 (2008).

8. Schneider, G. F. \& Dekker, C. DNA sequencing with nanopores. Nat. Biotechnol. 30, 326-328 (2012).

9. Malmstadt, N., Jeon, T. J. \& Schmidt, J. J. Long-lived Planar Lipid Bilayer Membranes Anchored to an In Situ Polymerized Hydrogel. Adv. Mater. 20, 84-89 (2008).
10. Shao, C., Sun, B., Colombini, M. \& DeVoe, D. L. Rapid Microfluidic Perfusion Enabling Kinetic Studies of Lipid Ion Channels in a Bilayer Lipid Membrane Chip. Ann. Biomed. Eng. 39, 2242-2251 (2011).

11. Portonovo, S. A. \& Schmidt, J. Masking apertures enabling automation and solution exchange in sessile droplet lipid bilayers. Biomed. Microdev. 14, 187-191 (2012).

12. Tsuji, Y. et al. Droplet based lipid bilayer system integrated with microfluidic channels for solution exchange. Lab Chip (2013).

13. Egorova, E. M., Chernomordik, L. V., Abidor, I. G. \& Chizmadzhev, Y. A. Liposome fusion with planar lipid membranes. Biofizika 26, 145-147 (1981).

14. Gutknecht, J. Inorganic mercury $(\mathrm{Hg} 2+)$ transport through lipid bilayer membranes. J. Membr. Biol. 61, 61-66 (1981).

15. Schadt, M. \& Haeusler, G. Permeability of lipid bilayer membranes to biogenic amines and cations: changes induced by ionophores and correlations with biological activities. J. Membr. Biol. 18, 277-294 (1974).

16. Pintschovius, J. \& Fendler, K. Charge Translocation by the $\mathrm{Na}+/ \mathrm{K}+-\mathrm{ATPa} e$ Investigated on Solid Supported Membranes: Rapid Solution Exchange with a New Technique. Biophys J 76, 814-826 (1999).

17. Jeon, T.-J., Malmstadt, N. \& Schmidt, J. J. Hydrogel-Encapsulated Lipid Membranes. J. Am. Chem. Soc. 128, 42-43 (2006).

18. Kang, X. F., Cheley, S., Rice-Ficht, A. C. \& Bayley, H. A storable encapsulated bilayer chip containing a single protein nanopore. J. Am. Chem. Soc. 129, 4701-4705 (2007).

19. Shim, J. W. \& Gu, L. Q. Stochastic sensing on a modular chip containing a singleion channel. Anal Chem 79, 2207-2213 (2007).

20. Funakoshi, K., Suzuki, H. \& Takeuchi, S. Lipid bilayer formation by contacting monolayers in a microfluidic device for membrane protein analysis. Anal Chem 78, 8169-8174, doi:10.1021/ac0613479 (2006).

21. Zagnoni, M., Sandison, M. E., Marius, P. \& Morgan, H. Bilayer lipid membranes from falling droplets. Anal. Bioanal. Chem. 393, 1601-1605 doi:10.1007/s00216 008-2588-5 (2009).

22. Bayley, H. et al. Droplet interface bilayers. Mol. Biosyst. 4, 1191-1208 (2008)

23. Ide, T., Kobayashi, T. \& Hirano, M. Lipid bilayers at the gel interface for single ion channel recordings. Anal Chem 80, 7792-7795 (2008).

24. Sarles, S. A., Stiltner, L. J., Williams, C. B. \& Leo, D. J. Bilayer formation between lipid-encased hydrogels contained in solid substrates. ACS Appl. Mater. Interfaces 2, 3654-3663 (2010).

25. Thompson, J. R., Heron, A. J., Santoso, Y. \& Wallace, M. I. Enhanced stability and fluidity in droplet on hydrogel bilayers for measuring membrane protein diffusion. Nano Lett. 7, 3875-3878 (2007)

26. Lu, X., Leitmannova Ottova, A. \& Tien, H. T. Biophysical aspects of agar-gel supported bilayer lipid nembranes: a new method for forming and studying planar bilayer lipid membranes. Bioelectrochem. Bioenerg. 39, 285-289 (1996).

27. Poulos, J. L. et al. Ion channel and toxin measurement using a high throughput lipid membrane platform. Biosens. Bioelectron. 24, 1806-1810 (2009).

28. Poulos, J., Portonovo, S., Bang, H. \& Schmidt, J. Automatable lipid bilayer formation and ion channel measurement using sessile droplets. J. Phys.: Condens. Matter 22, 454105 (2010).

29. Bamberg, E. \& Läuger, P. Channel formation kinetics of gramicidin A in lipid bilayer membranes. J. Membr. Biol. 11, 177-194 (1973).

30. Andersen, O. Ion movement through gramicidin A channels. Single-channel measurements at very high potentials. Biophys. J. 41, 119-133 (1983).

31. Mamonov, A. B., Kurnikova, M. G. \& Coalson, R. D. Diffusion constant of $\mathrm{K}^{+}$ inside Gramicidin A: A comparative study of four computational methods. Biophys. Chem. 124, 268-278 (2006).

32. McKemy, D. D., Neuhausser, W. M. \& Julius, D. Identification of a cold receptor reveals a general role for TRP channels in thermosensation. Nature 416, 52-58 (2002).

33. Peier, A. M. et al. A TRP channel that senses cold stimuli and menthol. Cell $\mathbf{1 0 8}$, 705-715 (2002).

34. Tsavaler, L., Shapero, M. H., Morkowski, S. \& Laus, R. Trp-p8, a novel prostatespecific gene, is up-regulated in prostate cancer and other malignancies and shares high homology with transient receptor potential calcium channel proteins. Cancer Res. 61, 3760-3769 (2001).

35. Zhang, L. \& Barritt, G. J. TRPM8 in prostate cancer cells: a potential diagnostic and prognostic marker with a secretory function? Endocr.-Relat. Cancer 13, 27-38 (2006).

36. Andersson, D. A., Chase, H. W. N. \& Bevan, S. TRPM8 activation by menthol, icilin, and cold is differentially modulated by intracellular pH. J. Neurosci. 24, 5364-5369 (2004).

37. Tsuruda, P. R., Julius, D. \& Minor, D. L. Coiled Coils Direct Assembly of a ColdActivated TRP Channel. Neuron 51, 201-212 (2006).

38. McKemy, D. D. Therapeutic potential of TRPM8 modulators. Open Drug Discov. J 2, 81-88 (2010).

39. $\mathrm{Hu}, \mathrm{H}$. Z. et al. 2-aminoethoxydiphenyl borate is a common activator of TRPV1, TRPV2, and TRPV3. J. Biol. Chem. 279, 35741-35748 (2004).

40. ChanTest, Stably Transfected Cell Line - Product Data Sheet hTRPM8-CHO http://www.chantest.com/media/cms/pdf/data_CT6193.pdf., Date of Access April 12013

41. Hamer, W. J. \& Wu, Y.-C. Osmotic coefficients and mean activity coefficients of uni-univalent electrolytes in water at 25 C. J. Phys. Chem. Ref. Data 1, 1047-1099 (1972). 
42. Hille, B. Ion Channels of Excitable Membranes. (Sinauer Associates, 2001).

43. Busath, D. D. et al. Noncontact Dipole Effects on Channel Permeation. I. Experiments with (5F-Indole)Trp13 Gramicidin A Channels. Biophys. J. 75, 2830-2844 (1998).

44. Kolb, H. A. \& Bamberg, E. Influence of membrane thickness and ion concentration on the properties of the gramicidin A channel Autocorrelation, spectral power density, relaxation and single-channel studies. $B B A$ Biomembranes 464, 127-141 (1977).

45. Zakharian, E., Cao, C. \& Rohacs, T. Gating of transient receptor potential melastatin 8 (TRPM8) channels activated by cold and chemical agonists in planar lipid bilayers. J. Neurosci. 30, 12526-12534 (2010).

\section{Acknowledgments}

We thank Dino Di Carlo, Takasi Nisisako, and Ahmad El-Arabi for consultation and Quincy Chen for assistance with chip fabrication.

\section{Author contributions}

J.S. conceived the study design and analyzed data. S.A., A.P., C.S. contributed to experiment design, performed experiments, and analyzed data. S.A. and A.P. contributed to device concept and fabrication. S.A., C.S. and J.S. drafted the paper. All of the authors discussed the results and edited the manuscript.

\section{Additional information}

Supplementary information accompanies this paper at http://www.nature.com/ scientificreports

Competing financial interests: The authors declare no competing financial interests.

How to cite this article: Acharya, S.A., Portman, A., Salazar, C.S. \& Schmidt, J.J.

Hydrogel-Stabilized Droplet Bilayers for High Speed Solution Exchange. Sci. Rep. 3, 3139; DOI:10.1038/srep03139 (2013).

\section{(c) (i) (2) This work is licensed under a Creative Commons Attribution-}

By Na NanCommercial-ShareAlike 3.0 Unported license. To view a copy of this license, visit http://creativecommons.org/licenses/by-nc-sa/3.0 